\title{
DEVELOPMENT AND VALIDATION OF A QUASI-DIMENSIONAL MODEL FOR (M)ETHANOL-FUELLED SI ENGINES
}

\author{
${ }^{1}$ Vancoillie, Jeroen ; ${ }^{1}$ Sileghem, Louis; ${ }^{1}$ Demuynck, Joachim; ${ }^{1}$ Verhelst, Sebastian \\ ${ }^{1}$ Ghent University, Belgium;
}

KEYWORDS - Alcohols, Spark Ignition Engine, Thermodynamic, Modelling

\begin{abstract}
Methanol and ethanol are interesting spark-ignition engine fuels, both from a production and an end-use point of view. Despite promising experimental results, the full potential of these fuels remain to be explored. In this respect, quasi-dimensional engine simulation codes are especially useful as they allow cheap and fast optimization of engines. The aim of the current work was to develop and validate such a model for methanol-fuelled engines. Several laminar burning velocity correlations and turbulent burning velocity models were implemented in a QD code and their predictive performance was assessed for a wide range of engine operating conditions.
\end{abstract}

The effects of compression ratio and ignition timing on the in-cylinder combustion were well reproduced irrespective of the employed $u_{l}$ correlation or $u_{t e}$ model. However, to predict the effect of changes in mixture composition, the correlation and model selection proved crucial. Compared to existing correlations, a new correlation developed by the current authors led to better reproduction of the effects of equivalence ratio and residual gas content and the combustion duration.

For the turbulent burning velocity, the models of Damköhler and Peters consistently underestimated the influence of equivalence ratio and residual gas content on the combustion duration, while the Gülder, Leeds, Zimont and Fractals model corresponded well with the experiments. The combination of one of these models with the new $u_{l}$ correlation can be used with confidence to simulate the performance and efficiency of methanol-fuelled engines.

INTRODUCTION - The use of sustainable liquid alcohols in spark-ignition engines offers the potential of decarbonizing transport and securing domestic energy supply while increasing engine performance and efficiency compared to fossil fuels thanks to a number of interesting properties [1,2]. The most significant interesting properties of light alcohols include:

- High heat of vaporization, which causes considerable charge cooling as the injected fuel evaporates

- Elevated knock resistance, which allows to apply higher compression ratios (CR), optimal spark timing and aggressive downsizing.

- High flame speeds, enabling qualitative load control using mixture richness or varying amounts of exhaust gas recirculation (EGR).

The potential of neat light alcohol fuels (methanol and ethanol) has been demonstrated experimentally in both dedicated and flex-fuel alcohol engines [1]. In dedicated alcohol engines high compression ratios enable peak brake thermal efficiencies up to $42 \%$, while throttleless load control using EGR allows to spread the high efficiencies to the part load regions $[1,3]$. In flex-fuel engines the $\mathrm{CR}$ is limited due to knock constraints associated with gasoline operation, but still relative power and efficiency benefits of about $10 \%$ and $\mathrm{NO}_{\mathrm{x}}$ reductions of 5-10 g/kWh can be obtained over the entire load range thanks to more isochoric combustion, optimal ignition timing and reduced flow and dissociation losses [1]. 
Despite these promising experimental results, the full potential of alcohol blended fuels and their impact on engine control strategies remain to be explored. Today these issues can be addressed at low cost using system simulations of the whole engine, provided that the employed models account for the effect of the fuel on the combustion process.

In this respect quasi-dimensional (QD) engine simulation codes are especially useful as they are well suited to evaluate existing engines, performing parameter studies and predicting optimum engine settings without resorting to complex multidimensional models [4]. At Ghent University, a QD code for the power cycle of hydrogen fuelled engines has been developed and validated during earlier work (GUEST: Ghent University Engine Simulation Tool) [5, 6]. The current work aims to extend this code to alcohol blended fuels and to add models predicting the gas dynamics, knock onset and pollutant formation in engines running on these fuels [7]. In this paper elements of this work pertaining to the power cycle simulation of engines running on neat methanol are presented.

\section{SIMULATION PROGRAM}

Framework and assumptions - The focus in this paper is the validation of the power cycle model and turbulent combustion models for engine operation on neat methanol. Also, the inhouse GUEST code was coupled to a commercial gas dynamics simulation tool during the current work, to enable simulation of the entire engine cycle (GT-Power [8]).

The current two-zone QD power cycle model was derived using several standard assumptions, as mentioned in $[4,5]$. The equations for the rate of change of the cylinder pressure $d p / d \theta$, burned and unburned temperatures, $d T_{b} / d \theta$ and $d T_{u} / d \theta$, are derived from conservation of energy. Additionally, a number of models and assumptions are necessary to close these equations:

- Heat exchange is calculated separately for the cylinder liner, cylinder head and piston based on an extension of the Woschni model discussed in [9]

- The CFR (Cooperative Fuel Research) engine used for validation of the simulation (see later) has a simple disc-shaped combustion chamber and ran at a fixed speed of $600 \mathrm{rpm}$. Therefore turbulence quantities are calculated using a very simple turbulence model based on measurements done in a similar engine [10]. The integral length scale $L$ is kept constant at $1 / 5$ of the minimum clearance height, and the rms turbulent velocity $u^{\prime}$ linearly decreases according to:

$u^{\prime}=u_{T D C}^{\prime}[1-0.5(\theta-360) / 45]$

Where $u_{T D C}^{\prime}$ is the rms turbulent velocity at top dead centre (TDC), taken to be 0.75 times the mean piston speed, $\theta$ is the crank angle (360 at TDC of compression).

- The mass burning rate is derived from a turbulent combustion model. The one used in this work is based on the entrainment framework, where the rate of entrainment of unburned gas into the flame front is given by

$\frac{d m_{e}}{d \theta}=\rho_{u} A_{f} u_{t e}$

Where $m_{e}$ is the entrained mass, $A_{f}$ is the mean flame front surface, and $u_{t e}$ is the turbulent entrainment velocity (see later). The mass entrainment into the flame front is then supposed to burn with a rate proportional to the amount of entrained unburned gas, with a time constant $\tau_{b}$ :

$\frac{d m_{b}}{d \theta}=\left(m_{e}-m_{b}\right) / \tau_{b}$

$\tau_{b}=C_{3} \lambda_{T} / u_{l}$ 
Where $C_{3}$ is a calibration constant, $u_{l}$ is the laminar burning velocity and $\lambda_{t}$ is the Taylor length scale, given by:

$\lambda_{t}=1.5 \mathrm{~L} / \sqrt{R e_{t}}$

$R e_{t}=u^{\prime} L / v_{u}$

Where $L$ is integral turbulent length scale and $v_{u}$ is the kinematic viscosity of the unburned gases. Equations (2) and (3) are used as a mathematical representation of the effects of a finite flame thickness [5].

- The quantities $p, T_{u}, T_{b}, m_{u}, m_{b}$, and $m_{e}$ are initialized as mentioned in [5].

- Gas properties are taken from the standard GT-Power libraries [8], supplemented with data for methanol from the chemical oxidation mechanism of Li et al. [11].

- A flame propagating after spark ignition is first only wrinkled by the smallest scales of turbulence. For the simulations done in this work, a flame development multiplying factor for the turbulent entrainment velocity was used, based on work by Dai et al. [12]:

$\left(1-e^{-r_{f} / r_{c}}\right)\left(\frac{r_{f}}{r_{c}}\right)^{1 / 3}$

Where $r_{f}$ is the flame radius and $r_{c}$ a critical flame radius given by $r_{f}=C_{1} L$

- For simplicity, blowby rates and the influence of crevice volumes have been neglected.

Turbulent burning velocity model - A turbulent entrainment velocity $u_{t e}$ is needed for closure of Equation (2). A number of turbulent burning models were selected and implemented in the form summarized below (for a full description of the models see the references or [6])

Damköhler [13]: $\quad u_{t e}=C_{2} u^{\prime}+u_{n}$

Gülder [14]: $\quad u_{t e}=0.62 C_{2} u^{\prime 0.5} R e_{t}^{0.25}+u_{n}$

Leeds "K-Le" [15]: $\quad u_{t e}=0.88 C_{2} u^{\prime}(\text { KaLe })^{-0.3}+u_{n}$

Fractals [16]: $\quad u_{t e}=u_{n} R e_{t}^{0.75\left(D_{3}-2\right)}$

Zimont [17]:

$$
D_{3}=\frac{2.35 C_{2} u^{\prime}}{u^{\prime}+u_{n}}+2.0 \frac{u_{n}}{u^{\prime}+u_{n}}
$$

Peters [18]:

$$
\begin{aligned}
& u_{t e}=C_{2} u^{\prime 0.75} L^{0.75} D_{T}^{-0.25}+u_{n} \\
& u_{t e}=0.195 C_{2} u^{\prime} D a\left[\left(1+\frac{20.52}{D a}\right)^{0.5}-1\right]+u_{n}
\end{aligned}
$$

Where $C_{2}$ is a calibration constant, $u_{n}$ is the stretched laminar burning velocity, $K a$ is the Karlovitz stretch factor [15], Da is the Damköhler number which is calculated using a laminar flame thickness based on the kinematic viscosity. An alternative is to use a flame thickness correlation based on chemical kinetics calculations [19].

Laminar burning velocity correlation - Turbulent burning velocity models need (stretched) laminar burning velocity data of the air/fuel/residuals mixture at the instantaneous pressure and temperature. This implies the need for either a library of stretched flamelets or a model for the effect of stretch. However, calculating the local flame speed from stretch-free data and a stretch model requires stretch-free data, naturally. As of today, there are insufficient data on stretch-free burning velocities at engine conditions, for any fuel. Stretch and instabilities hamper the experimental determination of stretch-free data at higher (engine-like) pressures [20].

The authors have worked on the laminar burning velocity of methanol and ethanol mixtures, compiling data from the literature [21] and looking at numerical [21] as well as experimental [22] means to determine a suitable laminar burning velocity correlation. A laminar burning 
velocity correlation has been determined based on chemical kinetics calculations [21]. This correlation was used for the current work. No stretch model has been implemented in the code as of yet, partly because of a lack of reliable data regarding the effect of stretch on methanol flames at engine-like conditions [23].

The form of the developed correlation is given by:

$$
u_{l}\left(\Phi, p, T_{u}, f\right)=u_{l 0}(\Phi, p)\left(\frac{T_{u}}{T_{0}}\right)^{\alpha(\Phi, p)} F\left(\Phi, p, T_{u}, f\right)
$$

Where $\phi, p, T_{u}$ and $f$ are the fuel/air equivalence ratio, pressure, unburned mixture temperature and molar diluents ratio respectively. $u_{l 0}$ is the laminar burning velocity at $T_{0}=300 \mathrm{~K} . u_{l 0}$ and $\alpha$ are third order polynomial functions of $\phi$ and $p$.

Published simulation work on methanol and ethanol fuelled engines often resorts to outdated laminar burning velocity correlations by Gibbs \& Calcote [24] (used in [25]), Metghalchi \& Keck [26] (used in [25], [27] and [8]) and Gülder [28] (used in [29] and [30]). Some authors [27] prefer the more recent correlation of Liao et al. [31], which basically has the same form:

$u_{l}=u_{l 0}\left(\frac{T_{u}}{T_{0}}\right)^{\alpha}\left(\frac{p}{p_{0}}\right)^{\beta}(1-\gamma f)$

Where $u_{l 0}$ is a second order polynomial of $\phi, \alpha$ and $\beta$ are first order function of $\phi$ and $\gamma$ is a constant.

Compared these older correlations, the form of Eq. (14) captures the strong interaction effects between equivalence ratio, pressure and temperature. E.g. the temperature and pressure effects are not assumed to be independent. The influence of residuals is incorporated into a separate correction term $F\left(\Phi, p, T_{u}, f\right)$ which is also a third order polynomial function of $\Phi, p, T_{u}$ and $f$. The term reflects the fact that the burning velocity is less sensitive to residual gases at higher temperatures. For a deeper discussion of the correlation and the polynomial functions, the reader is referred to [21].

In the light of the discussion below, Figure 1 and Figure 2 highlight the main differences between the different methanol laminar burning velocity correlations in terms of equivalence ratio and residual gas content.

Compared to older correlations of Gibbs \& Calcote [24], Metghalchi \& Keck [26], Gülder [28] and Liao et al. [31], the new correlation -Equation (14)- places the peak laminar burning velocity at a richer equivalence ratio and predicts a less steep decrease in $u_{l}$ for rich mixtures. As mentioned in [21] the correlation of Gibbs and Calcote [24] considerably overestimates $u_{l}$ due to uncertainties in the experimental method employed by these authors.

The residual gas correction term $F$ in the current correlation produces values comparable to other correlation developed for methanol, but its third order form avoids negative values for $F$ at very high diluents ratios $(f>0.5)$. The correlation of Rhodes and Keck [32] predicts a steeper drop in burning velocity in terms of diluents ratio, but was developed for indolene/air/diluents mixtures. 


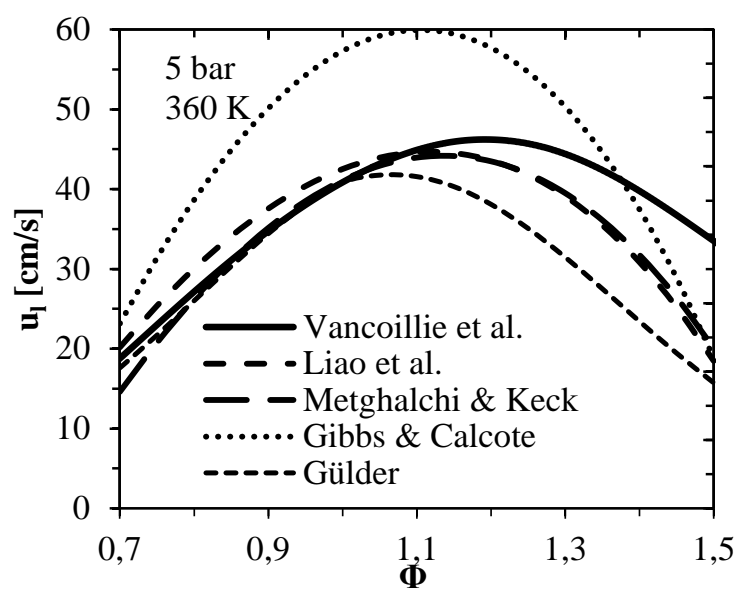

Figure 1: $u_{1}$ as a function of $\phi$ as predicted by several correlations

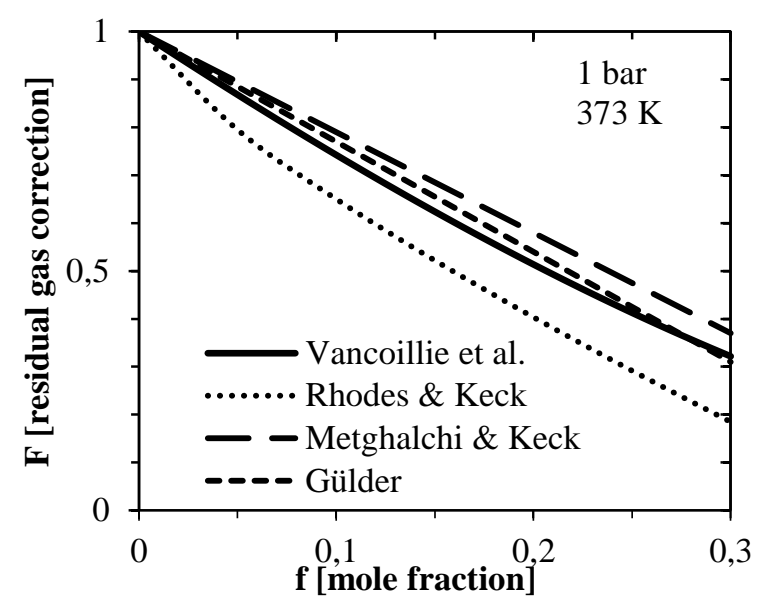

Figure 2: Comparison of different residual gas correction terms $\mathbf{F}$

\section{VALIDATION}

Engine - To validate the combustion models' predictive capabilities, a series of measurements were done on a port fuel injected single cylinder CFR engine, described in [33]. The main characteristics of this engine are summarized in Table 1.

Table 1: Characteristics of the single cylinder CFR engine

\begin{tabular}{|l|l|}
\hline Bore & $82.55 \mathrm{~mm}$ \\
\hline Stroke & $114.2 \mathrm{~mm}$ \\
\hline Swept Volume & $611.7 \mathrm{~cm}^{3}$ \\
\hline Geometry & Disc-shaped \\
\hline Speed & $600 \mathrm{rpm}$ \\
\hline IVO/IVC & $17^{\circ} \mathrm{CA}$ ATDC $/ 26^{\circ} \mathrm{CA}$ ABDC \\
\hline EVO/EVC & $32^{\circ} \mathrm{CA} \mathrm{BBDC} / 6^{\circ} \mathrm{CA}$ ATDC \\
\hline
\end{tabular}

The measurements comprise variable fuel/air equivalence ratio $\phi$, ignition timing (IT), compression ratio (CR) and throttle position (TP) (see Table 2). In order to allow distinction of the individual effects of these parameters, without resorting to a lot of one factor at a time sweeps, the experimental conditions have been chosen in such a way the Response Surface Methods can be applied to analyze the results [34]. This way, the resulting quantities of interest (e.g. IMEP, ignition delay) can be fit as a function of $\phi, C R$, IT and TP.

Table 2: Measurement conditions on the CFR engine

\begin{tabular}{|c|c|c|c|c|c|c|}
\hline No. & $\boldsymbol{\lambda}$ & $\boldsymbol{\phi}=\mathbf{1} / \boldsymbol{\lambda}$ & $\mathbf{C R}$ & $\mathbf{I T}\left[{ }^{\circ} \mathbf{c a ~ B T D C}\right]$ & $\mathbf{T P}\left[^{\circ}{ }\right]$ & EGR (\%) \\
\hline 1 & 1.00 & 1.00 & 9.0 & 15 & 75 & 6.1 \\
\hline 2 & 1.00 & 1.00 & 9.0 & 15 & 87 & 12.5 \\
\hline 3 & 1.00 & 1.00 & 9.0 & 5 & 75 & 6.3 \\
\hline 4 & 1.30 & 0.77 & 9.0 & 15 & 75 & 6.6 \\
\hline 5 & 1.15 & 0.87 & 8.5 & 20 & 81 & 10.0 \\
\hline 6 & 0.85 & 1.18 & 8.5 & 10 & 81 & 8.7 \\
\hline 7 & 1.15 & 0.87 & 8.5 & 10 & 69 & 5.8 \\
\hline 8 & 0.85 & 1.18 & 8.5 & 20 & 69 & 5.7 \\
\hline 9 & 1.15 & 0.87 & 9.5 & 10 & 81 & 8.5 \\
\hline 10 & 0.85 & 1.18 & 9.5 & 20 & 81 & 8.4 \\
\hline 11 & 1.15 & 0.87 & 9.5 & 20 & 69 & 5.7 \\
\hline 12 & 0.85 & 1.18 & 9.5 & 10 & 69 & 5.2 \\
\hline 13 & 0.70 & 1.43 & 9.0 & 15 & 75 & 6.2 \\
\hline 14 & 1.00 & 1.00 & 9.0 & 15 & 75 & 6.5 \\
\hline 15 & 1.00 & 1.00 & 9.0 & 25 & 75 & 6.6 \\
\hline 16 & 1.00 & 1.00 & 9.0 & 15 & 63 & 5.6 \\
\hline
\end{tabular}

Model setup - As the main focus of the current work was to evaluate combustion models, the employed engine model is limited to the closed part of the engine cycle (IVC to EVO). The 
initial conditions for mass fractions of air and fuel, the mean temperature and pressure at IVC are taken from the measurements. The residual gases (from the previous engine cycle) are estimated using a gas dynamics model of the entire intake and exhaust geometry.

Calibration - The calibration sets the coefficients for the heat transfer model, the flame development model $\left(C_{1}\right)$ and the turbulent burning velocity model $\left(C_{2}, C_{3}\right)$. For each model, the code has been calibrated at the first measurement condition in Table 2, as this condition is in the middle of the explored parameter space. The calibration constants are left constant for the other conditions.

The heat transfer calibration constant was set to 1.3 for all simulations, based on correspondence between the measured and predicted cylinder pressure during compression. The combustion model was calibrated by minimizing either the error in IMEP or the sum of squared differences between the measured and predicted normalized burn rate. These two methods lead to slightly different simulation results, as will be explained later. The measured burn rate was derived from the measured cylinder pressure by doing a reverse heat release analysis using the same cylinder model as used in the forward power cycle simulation [8]. The flame development constant $C_{1}$ is usually calibrated first in order to get a reasonable correspondence for the ignition delay. As mentioned in [5], increasing $C_{2}$ increases the mass entrainment rate, while increasing coefficient $C_{3}$ decreases the mass burning rate.

Consequently, both constants are simultaneously optimized using the Design of Experiments and Response Surface Methods embedded in GT-Power [8].

\section{RESULTS}

Comparison of laminar burning velocity correlations - A first part of the work consisted of comparing the predictive capabilities of different laminar burning velocity correlations for methanol. Three correlations are considered here: the correlation of Gülder [35], the correlation for methanol implemented in GT-Power, which is based on the correlation of Metghalchi and Keck [26] and the residual gas term of Rhodes and Keck [32], and the new correlation - Eq. (14) - developed by the current authors [21]. To minimize the influence of the turbulent burning velocity model, the Damköhler model was used with the same calibration constants for all simulations discussed in this section $\left(C_{1}=0.5, C_{2}=0.46, C_{3}=1\right)$.

The simulation results are synthesized into graphs showing the ignition delay $\Delta_{I G N}$ (defined as ${ }^{\circ} \mathrm{Ca}$ from $0 \%$ to $2 \%$ burned mass), the $10-90 \%$ burn time $\left(\Delta_{10-90 \%}\right)$ and the maximum cylinder pressure $\left(P_{M A X}\right)$. Figure 3 compares these quantities for variable compression ratio. Ignition delay slightly reduces at higher compression ratios. This is reproduced in by the model due to a reduced burn-up time constant $\tau_{b}$ at higher CR. This can be understood from looking at Equations (4) and (5). Increasing the CR decreases $L$ (because of smaller top dead center clearance) and increases $u_{l}$ (because temperature and pressure increase and residual gas content decreases). The experimental $10-90 \%$ burn times is almost independent of CR, whereas all models predict a slight increase at higher CR. Additional measurements at more extreme compression ratios could help to better understand the effect of $\mathrm{CR}$ on the main combustion.

Figure 4 compares $\Delta_{I G N}, \Delta_{10-90 \%}$ and $P_{M A X}$ for variable ignition timing. The ignition delay is barely influenced by spark timing. One might expect a slight reduction at more retarded spark timing due to the higher $u_{l}$ (higher $p, T$ ). However, in the models this effect is counteracted by the reduction in $u^{\prime}$ and corresponding increase in burn-up time constant $\tau_{b}$. The reduction in $u^{\prime}$ at more retarded ignition timing (see Equation (1)) is also responsible for the increase in $10-90 \%$ burn time, reflected in the measurements. 


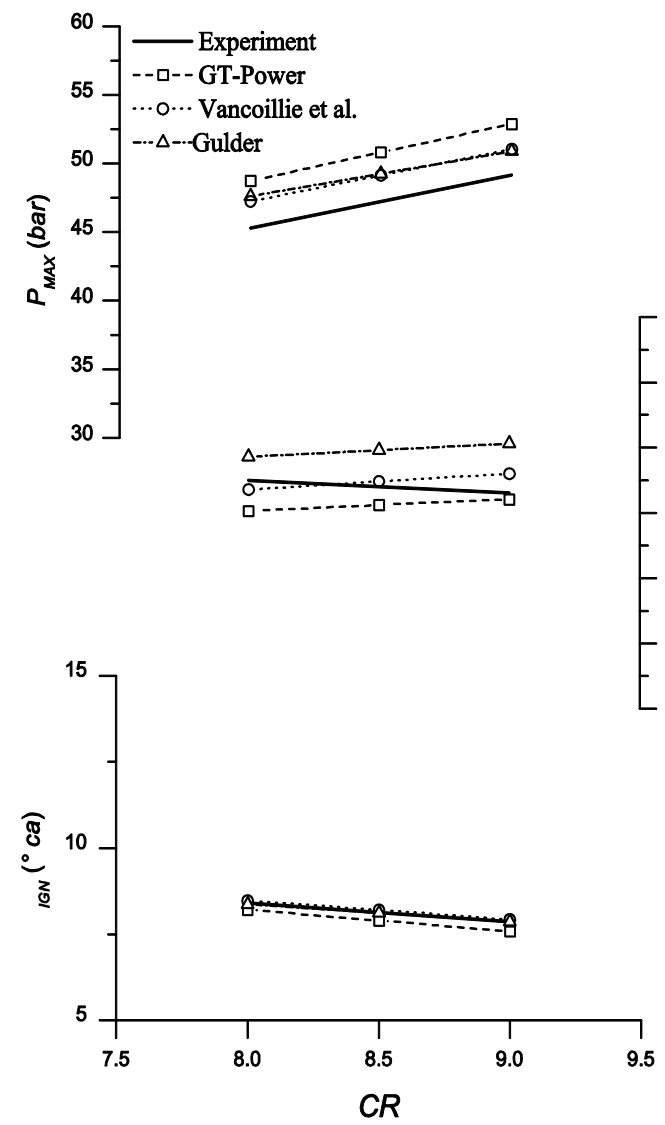

Figure 3: Comparison of $\boldsymbol{u}_{l}$ correlations for varying compression ratio

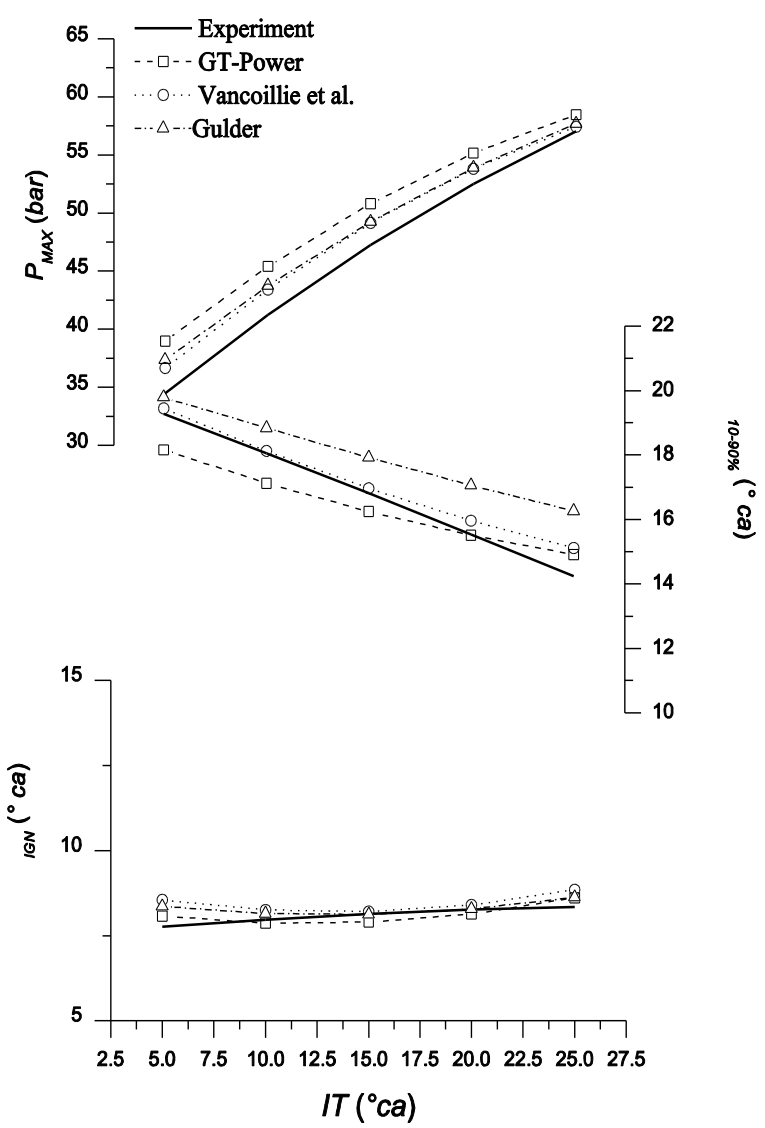

Figure 4: Comparison of $u_{1}$ correlations for varying ignition timing

Note that in all of the simulation results discussed in this paper, the maximum pressure is generally overpredicted. Possible reasons can be understood from looking at burn rates and corresponding pressure traces for condition 12 (Table 2) in Figure 5. When comparing the simulated burn rate (dashed line) with those derived from the experimental pressure traces (full line), it can be seen that the latter ones are truncated at the peak and exhibit a tail. This is probably caused by an accumulation of inaccuracies. Blow-by and fuel in the crevice volume are neglected. Also, an identical heat transfer coefficient is used for the compression, combustion and expansion, leading to an underprediction of the heat transfer during combustion. These inaccuracies could be cured by further model development, but do not impede the comparison of $u_{l}$ correlations and turbulent burning velocity models aimed at here. Also shown in Figure 5 are the laminar $\left(u_{l}\right.$ - full line) and turbulent ( $u_{t}$ - dashed line) contributions to turbulent entrainment velocity $u_{t e}$. This demonstrates the large share of the laminar burning velocity in the entrainment velocity
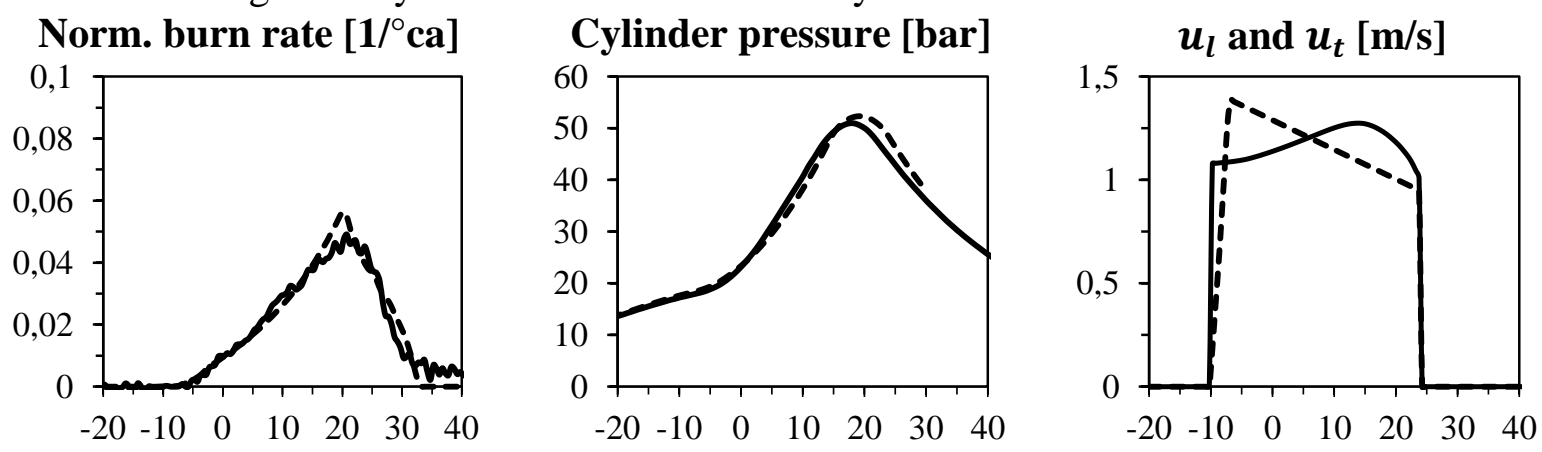

Figure 5: Experimental (full line) and simulated (dashed line) normalized burn rate and corresponding cylinder pressure. The rightmost figure shows the simulated laminar and turbulent contribution to $u_{t e}$ 
The predictive capabilities of the model for changes in CR and IT show little dependency on the employed laminar burning velocity correlation. As the laminar burning velocity groups the contribution of chemical kinetics to the combustion, its effects are most pronounced for changes in mixture composition.

Figure 6 displays the results for varying equivalence ratio $(\phi=0.77-1.43)$. For lean and rich mixtures, the ignition delay can be seen to be vastly overpredicted by traditional $u_{l}$ correlations. As the predicted ignition delay is mainly determined by the laminar burning velocity, this is a direct effect of the erroneous evolution of these correlations as a function of $\phi$ (see Figure 1). As the 10-90\% burn time is dominated by the turbulent combustion velocity, the difference between the correlations is less pronounced for $\Delta_{10-90 \%}$. Still, the results indicate a better performance for the new correlation.

Finally, Figure 7 shows the effect of throttle position. A more closed throttle $\left(90^{\circ}=\right.$ closed $)$ not only reduces the temperature and pressure of the cylinder charge, but also implies a higher residual gas percentage (see Table 1). The effect of TP on $\Delta_{I G N}$ is overpredicted by the GTPower correlation, indicating that the residual gas correction term of Rhodes and Keck produces a too steep decrease of $u_{l}$ as a function of diluents ratio (see Figure 2). For the main combustion duration $\Delta_{10-90 \%}$, however, this correlation leads to the best correspondence. The new correlation and the Gülder correlation result in an underprediction of the effect of TP on the main combustion. As we shall see in the next chapter, this is an artifact of the Damköhler turbulent burning velocity model, rather than an effect of the $u_{l}$ correlation.

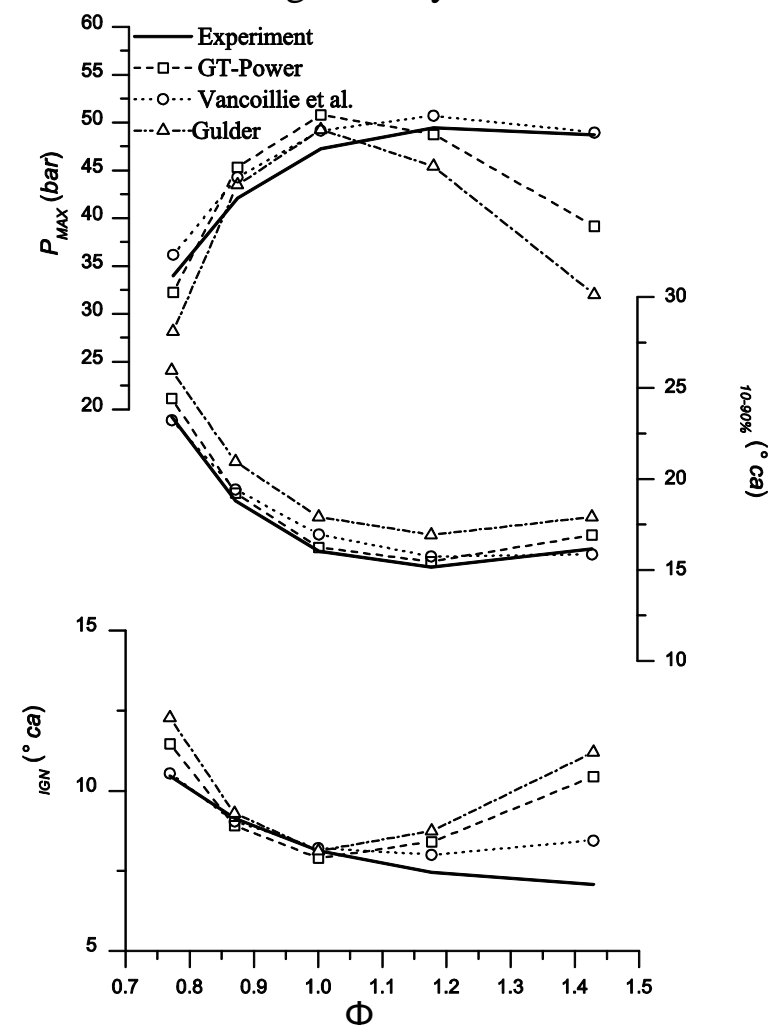

Figure 6: Comparison of $\boldsymbol{u}_{l}$ correlations for varying equivalence ratio

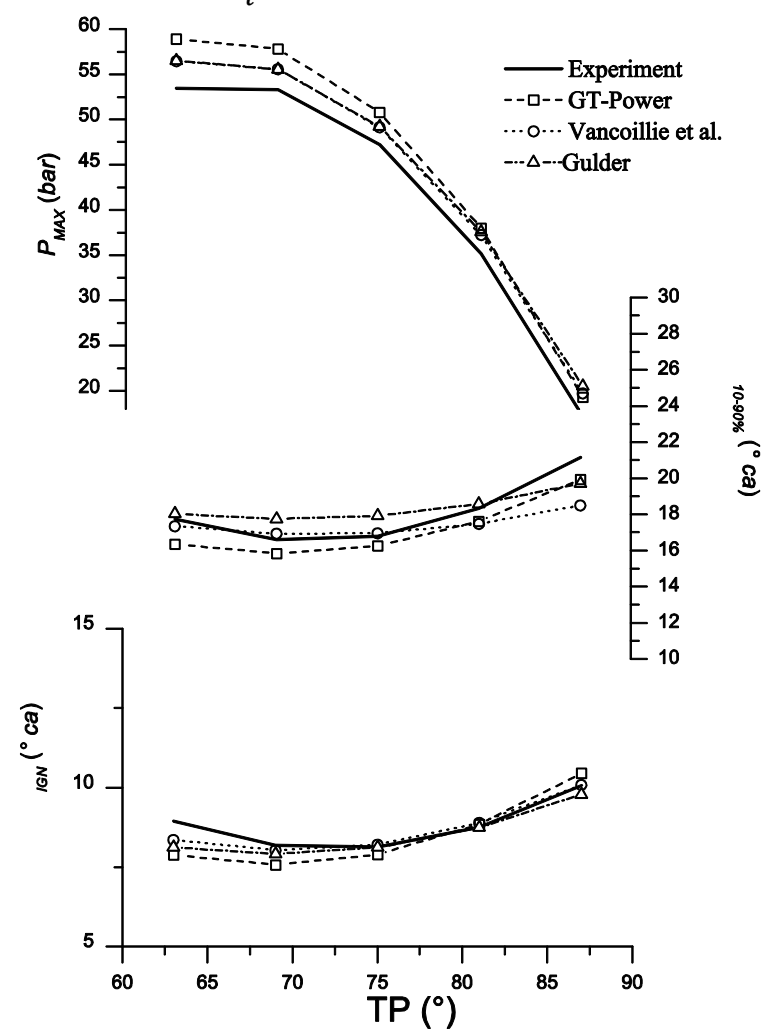

Figure 7: Comparison of $\boldsymbol{u}_{l}$ correlations for varying throttle position

Comparison of turbulent burning velocity correlations - In the second part of this work, the predictive performance of the different turbulent burning velocity models implemented in the code was compared. For all the simulations in this section, the new laminar burning velocity correlation was used (Eq. (14)). The calibration constants for the six turbulent burning velocity models considered here are summarized in Table 3. Note that the Peters and Fractals 
models have been calibrated for minimal IMEP error, whereas the other models have been calibrated for best correspondence with the measured burn rate. As can be seen from Figure 8 and Figure 9, the former method leads to better peak pressure correspondence, while the latter leads to a better prediction of the crank angle of maximum pressure $c a_{P \max }$.

Table 3: Calibration constants of turbulent burning velocity models

\begin{tabular}{|l|r|r|r|}
\hline Model & \multicolumn{1}{|c|}{$\boldsymbol{C}_{\mathbf{1}}$} & \multicolumn{1}{c|}{$\boldsymbol{C}_{\mathbf{2}}$} & \multicolumn{1}{c|}{$\boldsymbol{C}_{\mathbf{3}}$} \\
\hline Damköhler & 0.8 & 0.75 & 0.15 \\
\hline Gülder & 1 & 0.157 & 0.2 \\
\hline Fractals & 1 & 0.943 & 0.156 \\
\hline Zimont & 0.4 & 0.156 & 0.139 \\
\hline Leeds & - & 0.23 & 0.5 \\
\hline Peters & - & 0.44 & 0.78 \\
\hline
\end{tabular}

The results for varying CR and IGN are very similar to those of the Damköhler model (see Figure 5 and Figure 3), irrespective of the applied turbulent burning velocity model and are not repeated here.

Differences between the turbulent burning velocity models begin to appear when simulating changes in mixture composition. Figure 8 displays the $\Delta_{I G N}, \Delta_{10-90 \%}, P_{M A X}$ and $c a_{P \max }$ for varying throttle position. As mentioned before, a more closed throttle reduces the laminar burning velocity, leading to an increase in ignition delay, which is well predicted by all the models. However, the increased residual gas content also lengthens the main combustion period $\Delta_{10-90 \%}$ and the ability to predict this effect clearly is the criterion to distinguish between models. The models of Gülder, Fractals, Zimont and Leeds follow the trend observed in the measurements, while the Damköhler and Peters model predict almost no dependency on the throttle position. The results for maximum pressure and crank angle of maximum pressure show that the models that have been calibrated for minimal IMEP error indeed lead to a better peak pressure correspondence but more retarded location of peak pressure.

As explained in [5] the appearance of different model groups can by understood from rewriting the models' equations using the definitions of $R e_{t}, D a, K a$ and $L e$.

Damköhler: $\quad u_{t e} \sim C u^{\prime}+u_{n}$

Gülder: $\quad u_{t e} \sim C u^{\prime 0.75} u_{n}^{0.5} L^{0.25} v_{u}^{-0.25}+u_{n}$

Leeds "K-Le": $\quad u_{t e} \sim C u^{\prime 0.55} u_{n}^{0.6} L^{0.15} v_{u}^{-0.15} D_{T, u}^{-0.3} D_{M}^{0.3}+u_{n}$

Fractals:

$u_{t e} \sim C u^{\prime 0.25} u_{n} L^{0.25} v_{u}^{-0.26}+u_{n}$

Zimont:

$$
u_{t e} \sim C u^{\prime 0.75} u_{n}^{0.5} L^{0.25} D_{T, u}^{-0.25}+u_{n}
$$

Peters:

$$
u_{t e} \sim C u^{\prime}+u_{n}
$$

As opposed to the other models, the Damköhler and Peters model do not contain the laminar burning velocity $u_{n}$ in the turbulent contribution to $u_{t e}$. This explain why these models are less sensitive to changes in mixture composition, which are reflected in $u_{n}$.

The results for varying equivalence ratio in Figure 9 confirm the distinction between the Damköhler and Peters models, and the rest of the models. Compared to the other models, the ignition delay and 10-90\% burn times predicted by these models are less dependent on the changes in laminar burning velocity associated with the equivalence ratio. All models overpredict the ignition delay for rich mixture. During initial stages of combustion the flame resembles a stretched laminar flame. Rich methanol flames have been reported to exhibit negative Markstein numbers and cellular instabilities [36], which could result in a higher burning velocity during flame initiation. This effect cannot be capture by the simple burning velocity models considered here. 
The influence of equivalence ratio on the main combustion duration is slightly overestimated by the Gülder, Fractals, Leeds and Zimont models. Especially very lean and rich mixtures seem to burn faster than the models predict. The experimental work on methanol-air turbulent burning velocity of Lawes et al. [36] might give an explanation for this behavior. During contained explosions in a spherical bomb, these authors found that the ratio of $u_{t e} / u_{t}$ is slightly dependent on the equivalence ratio. The ratio was $50 \%$ higher at very lean $(\phi=0.7)$ and rich $(\phi=1.6)$ mixtures compared to the values at $\phi=1.0-1.3$. This is partly due to the preferential diffusion effects and diffusive thermal effects discussed by Lipatnikov and Chomiak [17] and cannot be captured by the models used here, which have a ratio $u_{t e} / u_{t}$ which is independent of the equivalence ratio.

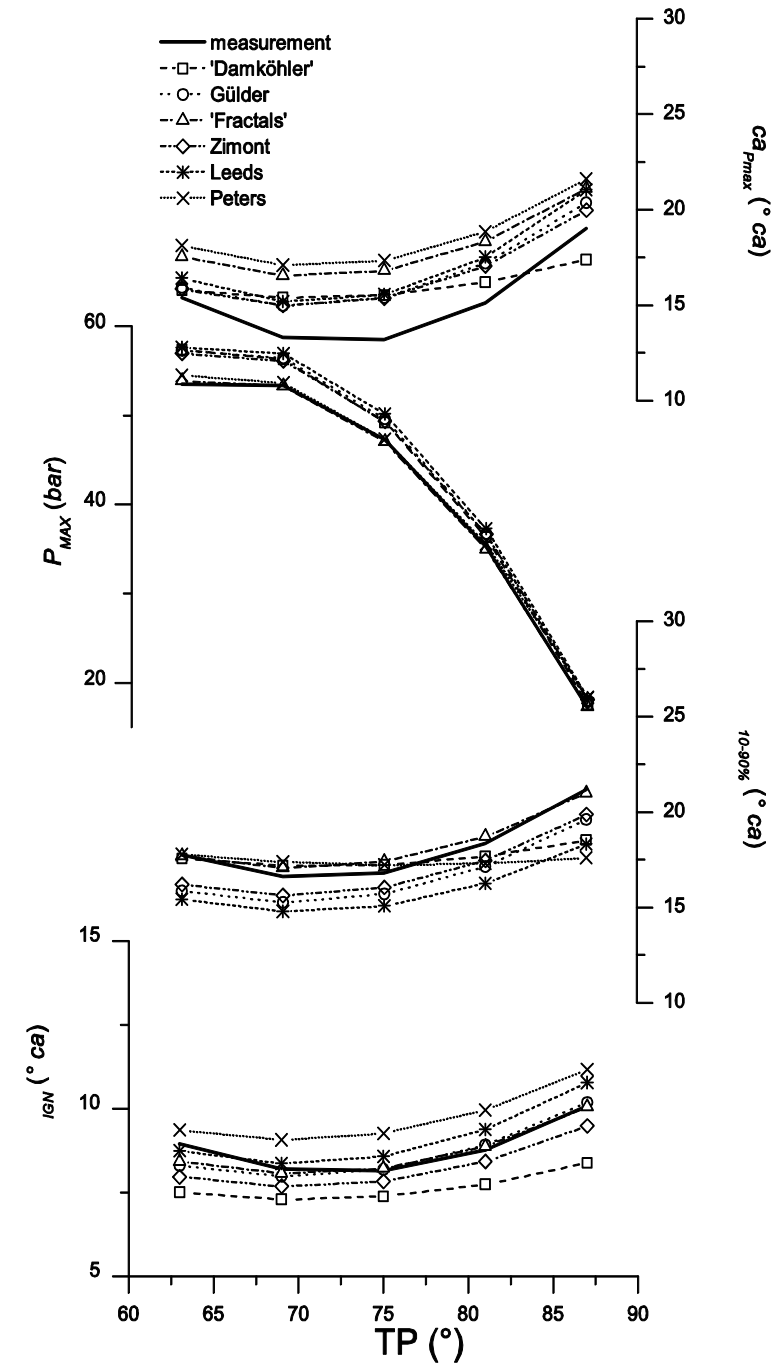

Figure 8: Comparison of turbulent burning velocity models for varying throttle position

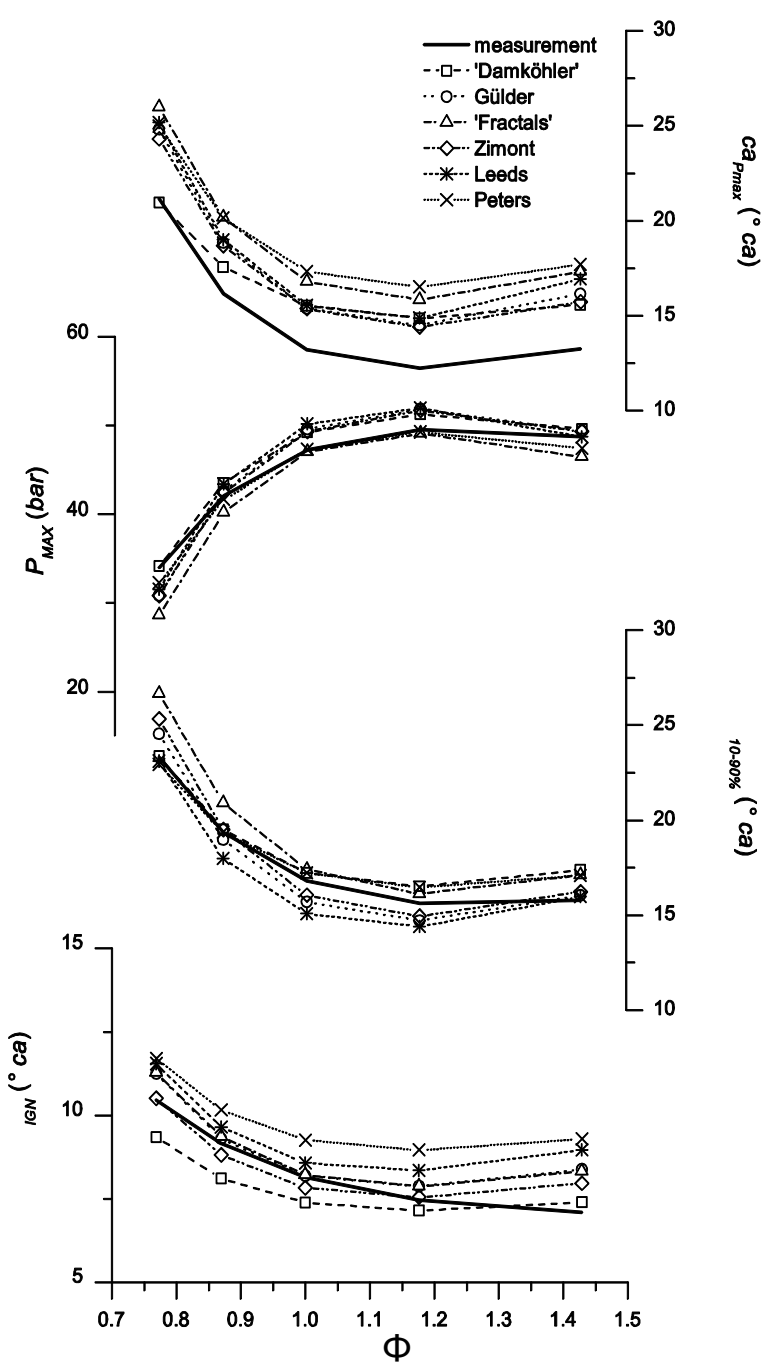

Figure 9: Comparison of turbulent burning velocity models for varying equivalence ratio

CONCLUSIONS - The focus of the current paper was the development and validation of a quasi-dimensional model for the combustion of neat methanol in spark-ignition engines. The predictive performance of several laminar burning velocity correlations and turbulent burning velocity models was assessed by considering a wide range of experimental conditions on a methanol-fuelled single cylinder engine.

The effects of compression ratio and ignition timing on the in-cylinder combustion were well reproduced by the simulation code, irrespective of the employed $u_{l}$ correlation or $u_{t e}$ model. To predict the effect of changes in mixture composition, the choice of laminar burning 
velocity correlation proved crucial. Compared to existing correlations, a new correlation developed by the current authors [21] led to considerably less overprediction of the combustion duration and ignition delay for rich and lean mixtures. Also the effect of residual gas content was better reproduced by the new correlation.

The ability to predict the effects of mixture composition was also clearly the criterion to distinguish between turbulent burning velocity models. The models of Damköhler and Peters consistently underpredicted the influence of equivalence ratio and residual gas content on the combustion duration, while the Gülder, Leeds, Zimont and Fractals model corresponded well with the experiments. The combination of one of these models with the new $u_{l}$ correlation can be trustfully used to simulate the performance and efficiency of methanol-fuelled engines and forms a solid base for further work regarding pollutant formation and knock modeling in alcohol engines.

ACKNOWLEDGEMENTS - J. Vancoillie and L. Sileghem gratefully acknowledge a Ph. D. fellowship of the Research Foundation - Flanders (FWO09/ASP/030 and FWO11/ASP/056). The authors would like to thank BioMCN for providing the bio-methanol used in this study.

\section{REFERENCES}

1. Vancoillie, J., J. Demuynck, L. Sileghem, et al., "The potential of methanol as a fuel for flex-fuel and dedicated spark-ignition engines", Applied Energy, 2012.

2. Pearson, R.J., J.W.G. Turner, M.D. Eisaman, et al., "Extending the Supply of Alcohol Fuels for Energy Security and Carbon Reduction", SAE paper no. 2009-01-2764, 2009.

3. Brusstar, M.J., M. Stuhldreher, D. Swain, et al., "High Efficiency and Low Emissions From a PortInjected Engine With Neat Alcohol Fuels", EPA VW 1.9 TDI measurements, SAE paper no. 2002-012743, 2002.

4. Verhelst, S. and C.G.W. Sheppard, "Multi-zone thermodynamic modelling of spark-ignition engine combustion - An overview", Energy Conversion and Management, 50(5), p. 1326-1335, 2009.

5. Verhelst, S. and R. Sierens, "A quasi-dimensional model for the power cycle of a hydrogen-fuelled ICE", International Journal of Hydrogen Energy, 32(15), p. 3545-3554, 2007.

6. Verhelst, S., "A study of the combustion in hydrogen-fuelled internal combustion engines. PhD thesis", Department of Flow, heat and combustion mechanics 2005.

7. Vancoillie, J. and S. Verhelst, "Modeling the combustion of light alcohols in SI engines: a preliminary study", presented at FISITA 2010 World Automotive Congress, Budapest, Hungary, 2010.

8. GammaTechnologies, "GT-Suite Version 7.0 User's Manual ", 2009.

9. Morel, T., C.I. Rackmil, R. Keribar, et al., "Model for heat transfer and combustion in spark ignited engines and its comparison with experiments", SAE paper no. 880198, 1988.

10. Hall, M.J. and F.V. Bracco, "A Study of Velocities and Turbulence Intensities Measured in Firing and Motored Engines", SAE paper no. 870453, 1987.

11. Li, J., Z.W. Zhao, A. Kazakov, et al., "A comprehensive kinetic mechanism for CO, CH2O, and CH3OH combustion", International Journal of Chemical Kinetics, 39(3), p. 109-136, 2007.

12. Dai, W., G.C. Davis, M.J. Hall, et al., "Diluents and Lean Mixture Combustion Modeling for SI Engines with a Quasi-Dimensional Model", SAE paper no. 952382, 1995.

13. Blizard, N.C. and J.C. Keck, "Experimental and Theoretical Investigation of Turbulent Burning Model for Internal Combustion Engines", SAE paper no. 740191, 1974.

14. Gülder, Ö.L., "Turbulent premixed flame propagation models for different combustion regimes", Symposium (International) on Combustion, 23(1), p. 743-750, 1991.

15. Abdel-Gayed, R.G., D. Bradley, and M. Lawes, "Turbulent Burning Velocities: A General Correlation in Terms of Straining Rates", Proceedings of the Royal Society of London. A. Mathematical and Physical Sciences, 414(1847), p. 389-413, 1987.

16. Wu, C.-M., C.E. Roberts, R.D. Matthews, et al., "Effects of Engine Speed on Combustion in SI Engines: Comparisons of Predictions of a Fractal Burning Model with Experimental Data", SAE paper no. 932714, 1993.

17. Lipatnikov, A.N. and J. Chomiak, "Molecular transport effects on turbulent flame propagation and structure", Progress in Energy and Combustion Science, 31(1), p. 1-73, 2005. 
18. Peters, N., "Turbulent Combustion", Cambridge University Press, Cambridge, 2000.

19. Vancoillie, J., J. Demuynck, J. Galle, et al., "A laminar burning velocity and flame thickness correlation for ethanol-air mixtures valid at spark-ignition engine conditions", Fuel, 2011.

20. Bradley, D., M. Lawes, K. Liu, et al., "Laminar burning velocities of lean hydrogen-air mixtures at pressures up to 1.0 MPa", Combustion and Flame, 149(1-2), p. 162-172, 2007.

21. Vancoillie, J., S. Verhelst, and J. Demuynck, "Laminar burning velocity correlations for methanol-air and ethanol-air mixtures valid at SI engine conditions", SAE paper no. 2011-01-0846, 2011.

22. Vancoillie, J., M. Christensen, E.J.K. Nilsson, et al., "Temperature Dependence of the Laminar Burning Velocity of Methanol Flames", Energy \& Fuels, 26(3), p. 1557-1564, 2012.

23. Zhang, Z., Z. Huang, X. Wang, et al., "Measurements of Laminar burning velocity and Markstein lengths for methanol-air-nitrogen mixtures at elevated pressures and temperatures", Combustion and Flame, 155(3), p. 358-368, 2008.

24. Gibbs, G.J. and H.F. Calcote, "Effect of Molecular Structure on Burning Velocity", Journal of Chemical I\& Engineering Data, 4(3), p. 226-237, 1959.

25. Brown, A.G., C.R. Stone, and P. Beckwith, "Cycle-by-cycle variations in spark ignition engine combustion - Part I: flame speed and combustion measurements and a simplified turbulent combustion model", SAE paper no. 960612, 1996.

26. Metghalchi, M. and J.C. Keck, "Burning velocities of mixtures of air with methanol, isooctane, and indolene at high pressure and temperature", Combustion and Flame, 48, p. 191-210, 1982.

27. Pourkhesalian, A.M., A.H. Shamekhi, and F. Salimi, "Alternative fuel and gasoline in an SI engine: A comparative study of performance and emissions characteristics", Fuel, 89(5), p. 1056-1063, 2010.

28. Gülder, Ö.L., "Laminar burning velocities of methanol, ethanol and isooctane-air mixtures", Symposium (International) on Combustion, 19(1), p. 275-281, 1982.

29. Bayraktar, H., "Experimental and theoretical investigation of using gasoline-ethanol blends in sparkignition engines", Renewable Energy, 30(11), p. 1733-1747, 2005.

30. Richard, S.B.S., "Modelling and simulation of the combustion of ethanol blended fuels in a SI engine using a OD coherent flame model", SAE paper no. 2009-24-0016 2009.

31. Liao, S.Y., D.M. Jiang, Z.H. Huang, et al., "Laminar burning velocities for mixtures of methanol and air at elevated temperatures", Energy Conversion and Management, 48(3), p. 857-863, 2007.

32. Rhodes, D.B. and J.C. Keck, "Laminar Burning Speed Measurements of Indolene-Air-Diluent Mixtures at High Pressures and Temperatures", SAE paper no. 850047, 1985.

33. Demuynck, J., M. De Paepe, H. Huisseune, et al., "Investigation of the influence of engine settings on the heat flux in a hydrogen- and methane-fueled spark ignition engine", Applied Thermal Engineering, 31(7), p. 1220-1228, 2011.

34. Box, G.E.P. and N.R. Draper, "Response Surfaces, Mixtures, and Ridge Analyses", John Wiley \& Sons, Hoboken, New Jersey, 2007.

35. Gülder, O.L., "Laminar Burning Velocities Of Methanol, Isooctane And Isooctane/Methanol Blends", Combustion Science and Technology, 33(1-4), p. 179-192, 1983.

36. Lawes, M., M.P. Ormsby, C.G.W. Sheppard, et al., "Variation Of Turbulent Burning Rate Of Methane, Methanol, And Iso-Octane Air Mixtures With Equivalence Ratio At Elevated Pressure", Combustion Science and Technology, 177(7), p. 1273 - 1289, 2005. 